\title{
New-York : symbole local et politique globale
}

New York : local symbol and global politics

\section{Sophie Body-Gendrot}

\section{(2) OpenEdition}

\section{Journals}

\section{Édition électronique}

URL : http://journals.openedition.org/conflits/744

DOI : $10.4000 /$ conflits.744

ISSN : $1777-5345$

\section{Éditeur :}

CCLS - Centre d'études sur les conflits lilberté et sécurité, L'Harmattan

\section{Édition imprimée}

Date de publication : 1 décembre 2001

Pagination : 77-96

ISBN : 2-7475-2227-X

ISSN : 1157-996X

\section{Référence électronique}

Sophie Body-Gendrot, « New-York : symbole local et politique globale», Cultures \& Conflits [En ligne], 44 | hiver 2001, mis en ligne le 22 mars 2006, consulté le 30 mars 2021. URL : http://

journals.openedition.org/conflits/744; DOI : https://doi.org/10.4000/conflits.744

Ce document a été généré automatiquement le 30 mars 2021

Creative Commons License 


\section{New-York : symbole local et politique globale}

New York: local symbol and global politics

Sophie Body-Gendrot

«Ecoute, mon pote, on a toujours su, les concepteurs de la déclaration des droits civiques euxmêmes le savaient, que la liberté est une chose fragile». William Brennan, juge à la Cour Suprême

A côté des débats contradictoires, utiles, profonds sur la signification des attentats du 11 septembre 2001 en relation avec la mondialisation, le « choc des civilisations » et la politique extérieure américaine, une des questions qu'on peut se poser sur leur conséquence est, à nouveau, la vulnérabilité des démocraties occidentales aux crises.

Cette vulnérabilité ne se manifeste pas seulement dans l'apparente facilité avec laquelle les attaques du 11 septembre ont été menées. Elle s'exprime surtout par la pression sur l'affect collectif - fierté nationale, malaise, peur - et par ses effets sur la facilité avec laquelle l'opinion dans ces démocraties semble accepter que des coupsde canif entament leurs principes fondamentaux.

Le monde entier - pas seulement occidental - risque d'être durablement déstabilisé par la chasse aux terroristes islamistes et à leurs sympathisants. Le risque vient de ce qu'une réaction de cette ampleur ne s'accommode pas de détails et que les catégorisations s'établissent sur des marqueurs simplistes liés au faciès et à l'appartenance. Il est, surtout, qu'une majorité de citoyens des pays occidentaux acceptent sans critique toutes les conséquences - labellisées « dommages collatéraux » des mesures anti-terroristes inévitables. Les dysfonctionnements d'une démocratie « disjonctive » ne peuvent que se trouver renforcés par les attentats du 11 septembre 2001 aux Etats-Unis. Cette expression ${ }^{1}$ signifie que dans les pays occidentaux, bien que le droit de vote et l'accès aux urnes soient des symboles forts de participation civique, ils demeurent insuffisants pour témoigner que la démocratie est mise en œuvre. Pour de nombreux citoyens résidant dans les villes, en effet, les conséquences de la mondialisation et de la migration, les processus de privatisation de l'espace, de la 
sécurité et de la justice, les discriminations, les inégalités socio-économiques, la nonnégociation et la violence limitent, déforment et érodent le concept de citoyenneté.

Si le terrorisme à l'origine des événements du 11 septembre atteint son but, c'est sans doute tout autant dans la destruction de bâtiments symboliques, le massacre de milliers d'innocents et l'éviscération d'une ville-monde que dans la mise à mal des pratiques démocratiques qu'acceptent les propres citoyens des pays dits libres.

De nouveaux ennemis « intérieurs »

Dans le climat sécuritaire qui s'est accentué de jour en jour depuis les attaques du World Trade Center et du Pentagone, on peut s'inquiéter dans la plupart des pays occidentaux, mais aux Etats-Unis plus particulièrement, du devenir des libertés constitutionnelles, de la liberté d'expression, de circulation, de la protection contre les excessives intrusions de l'Etat et du droit à une justice équitable. Va-t-on voir se profiler le spectre du maccarthysme à l'égard de catégories vulnérables et éternellement suspectes ? Depuis le 11 septembre, les plaintes pour discrimination dans les emplois en raison de la nationalité et de la religion émanent de tous les Etats et dans tous les secteurs. Près de cent personnes d'origine arabe se sont déjà portées devant les tribunaux américains. En France également on évoque «la galère des diplômés maghrébins " qui ne trouvent pas d'emplois après leurs stages et l'accroissement des discriminations. Depuis les attentats, l'idée d'une sorte de cinquième colonne, de réseaux terroristes diffus constitués par des ennemis infiltrés a focalisé l'attention sur les communautés arabo-musulmanes. Par voie de conséquence, les habituels exclus, Africains-Américains et Hispaniques se sont sentis plus «inclus » et ont multiplié les signes de leur loyauté... même envers police et pompiers, tandis que les autres subissaient la tyrannie de l'apparence. On peut être surpris que $71 \%$ des AfricainsAméricains se disent favorables au profiling à l'encontre des Arabes et que $64 \%$ d'entre eux réclament une carte d'identité pour les seuls musulmans, se démarquant des autres Américains qui se refusent à distinguer les seuls Arabes². Il n'y a là rien de surprenant. En période de crise, les symboles d'expression de loyauté envers la patrie se trouvent surinvestis. Tel président d'un conseil scolaire à Madison (Wisconsin) vient d'en être victime : il n'avait pas souhaité rendre le salut au drapeau obligatoire dans les écoles publiques de son district et il s'est vu contraint de démissionner sous la pression d'une hystérie collective. Désormais ici et là, des tests dans les pratiques quotidiennes vérifient qui est loyal, qui ne l'est pas, qui est un-American, etc.

Notre article poursuit un double objectif. D'une part, il vise à expliquer qu'après les attaques du 11 septembre 2001, l'échelon fédéral a donné dans un premier temps l'impression de flotter et que si le Président a su se ressaisir, des doutes ont subsisté sur l'efficacité de la CIA et du FBI dont l'architecture et les missions se sont révélées anachroniques. Par contraste, la manière magistrale dont l'ancien maire de New York, Rudolph Giuliani, a fait face à la catastrophe, en s'imposant aux divers échelons international, national et local - a démontré que l'efficacité au cours de cette première période venait de l'interaction d'un maire, de son équipe et des New Yorkais euxmêmes dans un pays valorisant l'autonomie locale.

D'autre part, l'article traite de la difficulté à trouver un équilibre entre sécurité et libertés. Il montre que les mesures destinées à rassurer l'opinion créent des clivages au sein de la démocratie américaine, les uns bénéficiant de protections constitutionnelles du fait de leur "américanité ", les autres étant soupçonnés de déloyauté. Il apparaît qu'avec l'option militaire, des causes profondes de l'attaque ne sont traitées ni à 
l'échelon mondial par une révision de la politique extérieure des Etats-Unis, ni au sein de la politique intérieure des Etats-Unis.

Un système de renseignement peu efficace et dispersé

Le 11 septembre 2001, les Etats-Unis ont subi la pire attaque de leur histoire, le nombre des victimes a dépassé celui de Pearl Harbor, correspondant à un dixième des morts du Vietnam - et le pays saisi d'effroi a cédé à une totale sidération. Les yeux se sont d'abord tournés vers le Président, puis plus tard, vers les agences fédérales chargées de la sécurité et accusées de n'avoir rien vu venir en dépit de leurs cent mille agents et du budget annuel de trente milliards de dollars dont elles disposent, budget excédant ceux de la défense nationale dans tous les pays de monde, à l'exception de six ${ }^{3}$.

Le problème tient d'abord à la fragmentation du système d'intelligence. Il ne compte pas moins de treize agences affiliées à cinq ministères. Le fonctionnement du centre du contre-terrorisme de la CIA créé en 1986 n'a cessé de se heurter à des frictions institutionnelles, à des rites de vérification, à des soupçons de déloyauté. Il ne peut, selon la loi, opérer sur le territoire des Etats-Unis. On a relevé une véritable aversion de la part de certaines agences à partager le moindre renseignement. La bureaucratie a envahi tous les organes de sécurité. En dépit des efforts de George Tenet, directeur du centre, celui-ci ne possède toujours pas de base de données performante sur les terroristes repérés comme tels et il n'a pas l'autorité nécessaire pour obliger les services à coopérer. La CIA et la National Security Agency (NSA) communiquent avec le bureau du Président, ce qui n'est pas le cas du FBI et des services d'immigration. La NSA aussi bien que l'agence chargée de l'imagerie satellitaire et des interceptions électroniques ne rendent pas de comptes à $\mathrm{M}$. Tenet, elles dépendent comme la plupart des services de contre-espionnage du Pentagone. Or le Pentagone semblait manquer au cours de ces dernières années à la fois de linguistes pour traiter les informations venues des conversations enregistrées dans les pays "voyous " et d'experts pour décrypter les photos des satellites. Il a de plus négligé, depuis la présidence de J. Carter, le renseignement humain pour se consacrer à l'anticipation d'une guerre futuriste à haute technologie. Le HUMINT (human intelligence) est devenu anémique tandis que gonflaient le MASINT (measurement and signature intelligence), l'ELINT (electronic intelligence) ou le SIGINT (signals intelligence). Certes, le budget supplémentaire d'un milliard de dollars alloué par le Congrès à l'Intelligence Service permettra de recruter de nouveaux agents, arabisants ou autres et de les former à la politique comparée. Mais il sera plus difficile de recruter et de former des agents de terrain, après des années de négligence.

Quant au FBI, 28000 agents des 56 bureaux dispersés à travers le pays ont reçu du Ministre de la Justice John Ashcroft et du directeur du FBI, Robert Mueller III, l'ordre de se concentrer exclusivement sur la prévention du terrorisme, alors qu'ils traitaient jusqu'alors des enlèvements, des attaques de banques, des tueurs en série, du contreespionnage et d'autres tâches plus prosaïques. Pourtant, même après cette redéfinition des missions, les résultats tardant à venir, en mai 2001, seuls $24 \%$ des Américains avaient une opinion favorable du FBI (contre $43 \%$ un an plus tôt) ${ }^{4}$. Ce n'est pas l'après-11 septembre qui modifiera ces perceptions: sur 1100 suspects arrêtés en septembre et en octobre, moins d'une douzaine avaient, semble-t-il, un lien avec l'attaque du World Trade Center. Devant l'indignation de quelques observateurs, le ministre de la Justice a rétorqué que s'il en était ainsi, désormais le nombre de suspects détenus ne serait plus communiqué à la presse. Qui sont-ils ? Combien d'entre eux sont 
des témoins ou des complices? Où sont-ils détenus? Quelles sont leurs nationalités? Sur quels chefs d'accusation les a-t-on arrêtés? Combien d'entre eux ont-ils été relâchés ? Outre l'enquête difficile à mener sur les lettres contaminées, le FBI a attribué son inefficacité aux contraintes légales qui lui avaient été imposées au cours des dernières années et qui l'empêchaient de pouvoir mener des interrogatoires musclés. Sauf à extrader les suspects dans un pays (sous-entendu voyou) aux méthodes plus expéditives, les agents se sont plaints de ne pouvoir aboutir, rappelant en écho le discours de la CIA sur les interdictions d'assassinats politiques et de fréquentations criminelles que lui avait imposé le Congrès (un éditorialiste de Newsweek, J. Alter, pourtant connu pour ses positions progressistes, n'a pas craint d'évoquer la torture "pour enfin faire décoller l'enquête sur le plus grand crime de l'histoire américaine »). Sans doute ces griefs sur la limitation des méthodes imposées par une démocratie sont-ils des écrans de fumée destinés à occulter l'inadaptation des agences fédérales de renseignement confrontées à un contexte de défi radicalement nouveau et à des adversaires énigmatiques qu'elles ont été mal préparées à affronter. Des équipes atypiques seraient-elles mieux capables de travailler sur des logiques de réseaux terroristes?

Le patriotisme comme recherche d'unité

Le public américain quant à lui, pris dans le maelström de ses émotions, a désespérément besoin de faire confiance à la compétence du gouvernement fédéral et à son chef symbolique ainsi qu'à son honnêteté. Il se bricole du sens à coups d'informations et de rumeurs circulant par capillarité. Il s'engage et se désengage selon des processus qui tiennent autant à la place de la violence dans la culture que de l'identification aux victimes ${ }^{5}$. Aujourd'hui, un sondage du 30 novembre $2001^{6}$ relatif à la législation anti-terroriste (Patriot Act du 12 octobre 2001) sur le homeland securityet àl'Executive Orderdu Président Bush révèle des réactions consensuelles et le brouillage des frontières entre sécurité extérieure et sécurité intérieureen un seul et même combat :

90\% des Américains approuvent les opérations militaires en Afghanistan (84\% des femmes et $77 \%$ des progressistes) et $89 \%$ soutiennent le Président, pourcentage inchangé depuis deux mois.

78\% des Américains seraient d'accord pour que les troupes américaines renversent Saddam Hussein.

Quatre Américains sur cinq sont prêts à sacrifier des libertés pour une plus grande sécurité.

$77 \%$ approuvent la création d'une carte d'identité pour tous.

$64 \%$ soutiennent les tribunaux militaires d'exception.

Trois Américains sur quatre disent que le gouvernement est dans son droit lorsqu'il met sur écoute les conversations ou ouvre le courrier des avocats aux détenus soupçonnés de terrorisme.

$79 \%$ d'entre eux approuvent les interrogatoires de 5000 jeunes hommes de 18 à 35 ans arrivés depuis deux ans du Moyen Orient et aux Etats-Unis avec des visas temporaires.

Neuf Américains sur dix trouvent que les Etats-Unis ont raison de détenir en prison ad infinitum plus de 600 étrangers ayant violé les lois d'immigration?

Ces opinions indiquent que les droits ne sont pas universels et que le profiling de personnes à risques est acceptable en période de guerre pour le peuple américain. Le 
renouveau du patriotisme est perceptible à l'auto-censure généralisée. Erreurs, reculs, incapacité du gouvernement fédéral à anticiper les événements sont acceptés pour autant que l'opinion ait la conviction que les interventions militaire, politique, diplomatique procèdent dans le bon sens et qu'à la différence de la guerre du Vietnam, il n'y ait pas dissimulation. Or là est le dilemme présidentiel : comment être efficace tout en communiquant sur les manœuvres poursuivies? Donald Rumsfeld, secrétaire d'Etat à la défense, a autorisé le Pentagone à «mentir » et le Président a ajouté qu'il n'était pas question de mettre les renseignements sur la place publique. Les Etats-Unis ne se ligotent-ils pas du fait de leurs ambiguïtés et de leurs ambivalences envers l'utilisation de leur puissance militaire ? Par contraste avec les débats et controverses soulevés par les décisions prises à l'échelon fédéral, la sphère locale apparait plus compréhensible, bien qu'elle soit, elle aussi, fragmentée par des enjeux de pouvoir relatifs à la sécurité.

New York, ville de tous les dangers

$\mathrm{Au}$ pied des tours disparues de Manhattan, dans South Ferry Park, un monument poignant en bronze évoque les marins disparus lors de la guerre d'Indépendance. Sur une barque de fortune, alors que tout s'effondre autour des pêcheurs, un homme se dresse, prêts à conjurer le destin. On ne peut qu'être frappé par le parallèle offert entre les circonstances présentes, ce monument et la nouvelle d'Edgar Poe sur la chute des pêcheurs dans le maelström. Norbert Elias propose une interprétation relative au statut des hommes en danger, groupes ou individus,empêtrés dans l'adversité les uns avec les autres ou avec eux-mêmes, de manière telle qu'il leur est difficile de se soustraire à cet enchevêtrement ${ }^{8}$. Un niveau élevé d'effroi trouve sa contrepartie, suggère-t-il, dans une forte charge imaginative liée aux représentations des périls. Celle-ci mène à la reproduction de modes de pensée plus tournés vers l'imaginaire que vers le réel, " corps à corps figé ", double contrainte, désignant de tels emmêlements. Toutefois si, plutôt que se recroqueviller sur sa peur comme le fait le jeune pêcheur, l'homme contrôle la violence de ses propres affects et procède à une évaluation réaliste du processus selon lequel l'entonnoir du maelström aspire les hommes et les biens, peutêtre pourra-t-il échapper à l'anéantissement.

Rudolph Giuliani, héros tragique

Au cours du mois d'août 2001, un sondage exprimait un niveau de satisfaction très élevé des New Yorkais à l'égard de leur maire, maire en partance puisque, selon la loi, il ne pouvait se présenter à un troisième mandat. Dans ce sondage, ils n'étaient plus qu'un tiers à vouloir déménager (contre $60 \%$ dix ans plus tôt), et un quart seulement à penser que les choses allaient empirer dans la ville au cours des dix à quinze années à venir ${ }^{9}$. Bref, il n'était plus nécessaire d'avoir un maire à stature de géant pour gérer la ville.

Or aujourd'hui, New York a besoin d'un tel maire. Un maire à la Colin Powell, figure d'autorité paternelle, inspirant confiance, sachant apaiser les inquiétudes et les peurs. Le désastre du World Trade Center est colossal: il accueillait 350 sociétés, 50000 salariés, une gare souterraine, plusieurs lignes de métro, un centre commercial. Avec la destruction des deux tours de cent dix étages, construites entre 1966 et 1973, ce sont 200000 tonnes d'acier et 413000 tonnes de béton qui ont été englouties d'une rive à l'autre de la pointe sud de Manhattan surnommée désormais ground zero - le point zéro - des milliers d'emplois qui ont disparu et des faillites qui sont annoncées ${ }^{10}$. Face à cette catastrophe, Rudolph Giuliani, tel le marin de la barque du South Ferry Park, s'est dressé et s'est imposé tant sur le plan international que national et local. Son mélange de stoïcisme et d'empathie en a fait un personnage aux dimensions héroïques, 
provoquant une admiration spontanée qui, jusqu'alors lui avait fait défaut. Il a été l'indispensable homme de la situation que le Washington Post a comparé à un "Churchill en casquette de baseball ». Il a su trouver pour ses concitoyens les mots justes, éclipsant pour un temps en popularité le président des Etats-Unis qui tardait à se rendre sur le site dévasté des tours « martyrisées » ${ }^{11}$.

La carrure internationale du maire a très vite émergé, comme le montrent deux exemples. Lorsqu'il s'est agi d'envoyer un message international de soutien à l'Amérique par le truchement des médias internationaux, Jacques Chirac ne s'y est pas trompé : c'est avec le maire de New York et non avec le Président W. Bush qu'il a choisi de survoler le site des Twin Towers en hélicoptère. De nombreux chefs d'Etat ont accompli la même démarche, à la fois pour redorer leur image et pour rendre hommage au maire. Par ailleurs, celui-ci a été le premier maire de la ville à se rendre aux Nations Unis et à s'adresser longuement aux délégués comme s'il était le chef d'une nation en guerre. "Ces attentats ne laissent aucune part à la neutralité », a-t-il souligné. "La preuve du mépris qu'entretiennent les terroristes à l'égard de la vie et du concept de paix gît sous les décombres du World Trade Center; à moins de trois kilomètres de l'endroit où nous sommes aujourd'hui réunis ».

Nationalement, lorsqu'il a reçu une centaine de représentants du Congrès devant les squelettes des Tours Jumelles, son discours a habilement mis en perspective la catastrophe économique, induite par la disparition de milliers d'emplois et de revenus, par rapport à l'économie nationale. Il a évoqué les entreprises détruites comme essentielles à l'économie américaine et comme reflets de sa performance. Il a su émouvoir l'ensemble du pays au point qu'un journal satirique ne craignait pas de titrer: "Le reste du pays éprouve temporairement de la compassion pour New York». De nombreuses villes ont en effet offert de porter matériellement secours à la ville, des milliers de bénévoles sont accourus de tous les Etats et les actes de solidarité ont été innombrables entre les habitants de la côte voisine du New Jersey et les New Yorkais, captifs de l'enceinte du World Trade et transportés par bateau dans l'Etat voisin pour y recevoir les premiers secours.

R. Giuliani possédait déjà un bilan local très solide à son actif avant la catastrophe. Bénéficiant d'une conjoncture économique exceptionnellement florissante, il avait contribué à ce que, grâce à une habile stratégie de marketing, New York acquiert la réputation d'une ville-monde, c'est à dire d'un lieu ex-territorialisé où flux et réseaux s'entremêlaient pour accumuler richesses, concentration de populations, de pouvoirs et de symboles. Le maire avait simplifié et assaini le budget et en rendant les rues sûres grâce à une réforme de la police locale de grande ampleur, entreprise alors que le nombre d'homicides annuels avoisinait 2200 (en 1992), il avait rassuré les investisseurs, les entrepreneurs, les habitants et les touristes ${ }^{12}$. Giuliani a créé une exigence d'ordre et de protection et le marché a fixé les normes, la condition des investissements, le type d'architecture sécuritaire et a transformé des quartiers à haute valeur ajoutée en espaces défensifs. Aujourd'hui le nombre d'homicides est tombé à 630 , ils ont baissé de $66 \%$ et ils ont continué à chuter au cours des semaines qui ont suivi l'attaque du 11 septembre pour remonter brusquement ensuite. Les larcins de grande ampleur ont diminué de $73 \%^{13}$. L'absence d'agressions brutales après le 11 septembre peut s'expliquer de plusieurs façons: d'abord le terrorisme a affecté le moral de tous les habitants, y compris celui de délinquants potentiels; ensuite, les minorités raciales les plus exclues ont cessé de se sentir ostracisées, puisque désormais 
les groupes terroristes sont bien plus stigmatisés qu'elles. L'attaque a réduit les clivages raciaux traditionnels, les minorités se sont senties solidaires, « américaines ", soumises aux mêmes menaces que tout Américain. Ajoutons que, compte tenu de la saturation de l'espace public par les forces de l'ordre - locales, subnationales et fédérales - il est devenu plus malaisé de poursuivre une carrière criminelle sur la voie publique, sauf à faire partie du crime organisé qui, comme son nom l'indique, est soupçonné d'avoir rapidement volé et expatrié 250 tonnes de ferraille provenant des ruines du World Trade vers deux sites du New Jersey et vers un autre sur Long Island. La reprise des homicides qui a affecté d'autres villes n'a pas touché New York et, selon les premières estimations, la criminalité aurait encore baissé de 12\% au cours de l'année 2001.

L'ancien maire de New York a su trouver les mots justes, se faisant l'écho de la philosophe S. Weil qui voyait dans la destruction de Troie la plus grande calamité qui puisse arriver à l'humanité : la destruction d'une cité, c'est à dire la capacité à vivre et à agir ensemble.

Les ressources d'une culture civique

De nombreux New Yorkais se sont sentis unis par l'admiration portée à des hommes ordinaires qui, tels les pêcheurs pris dans le maelström, ont fait face à la catastrophe : il s'agit des pompiers de New York, "nouveaux prêtres de ce pays " selon les uns, "nouveaux sex-symbols » selon les autres ${ }^{14}$. Le cadrage dominant par les médias pendant les dix jours de deuil qui ont suivi l'événement, s'est conformé au scénario du Bien contre le $\mathrm{Mal}$ : à ben Landen, figure emblématique du mal, et à ses complices les talibans, a été opposée la figure des sauveurs, les pompiers, au dévouement sans limite au point de laisser leur vie dans les décombres ${ }^{15}$. Autour du périmètre du "point zéro", sur les murs, tout au long des rues, ont fleuri des ex-votos parsemés des clichés familiers des pompiers en famille auxquels se relient non seulement les proches mais les visiteurs $d u$ monde entier soucieux de s'identifier à des symboles forts. Ils exaltent virilité et valeurs héroïques; mais cette lecture gomme les visages des femmes de cultures diverses, occupées dans les tours, en bas de la chaîne de montage mondiale, et aujourd'hui disparues ou sans emploi. Les médias proposent constamment des raccourcis et des symboles forts, destinés à susciter des réactions émotives et patriotiques, ce qui n'incite pas à comprendre des phénomènes plus complexes et contradictoires, in the making, qui demanderaient plus de temps et de réflexion. L'Institut de politique fiscale estime que 48000 des 80000 emplois qui auront été perdus par New York à la fin de l'année représentent de petits salaires d'environ 23000 dollars, soit moins de la moitié du salaire médian de 58000 dollars ${ }^{16}$. C'est moins le capitalisme et Wall Street qui ont été atteints par les terroristes qu'un groupe bien différent, composé de blanchisseuses, cuisinières, chauffeurs de taxi, guichetiers...

La culture civique new yorkaise reste une ressource dominante qui permettra à la ville de rebondir comme elle a toujours su le faire en temps de crise. Les New Yorkais ont compris à quel point les relations humaines, l'amitié et la simple survie leur étaient devenues précieuses. Ebranlés en profondeur par le choc du 11 septembre, leur réaction a moins été de demander l'aide de l'Etat que de se prendre en charge, de s'organiser, de participer. On peut citer de nombreux témoignages de cet activisme civique qui ne date pas d'hier et de cette énergie positive ainsi succinctement évoquée : "On ne savait pas quoi faire. Bien que quadragénaire, je suis vigoureux... J'ai voulu y aller, aider moi aussi à déblayer un peu... On a envie de passer devant toutes les casernes de pompiers et tous les postes de police pour serrer la main de ces types... Nous avons déposé des vêtements, des 
gants et d'autres affaires... Peut-être que dans quelques jours, quand certains de ceux qui travaillent là-bas seront vraiment fatigués, on permettra à des gens comme moi d'apporter leur aide. Je veux me rendre utile $»^{17}$. Les barrières de classe, de genre, de race ont été pour un moment levées.

$<$ !--SPIP--> Un avenir en sursis<//r2r:section1>

New York va-t-elle connaître les pages les plus sombres de son histoire dans les mois à venir? Sur ce point les avis sont partagés. Toutes les catégories socio-économiques vont être affectées par le déficit de la ville que l'on évalue entre 4 et 6 milliards de dollars (sur un budget annuel de 40 milliards de dollars), le déficit de l'Etat de New York étant évalué à 3 milliards de dollars. Comme il sera très difficile de couper dans les dépenses liées à la sécurité publique, aux salaires des employés municipaux, au fonctionnement des écoles publiques (dans un piètre état) et des hôpitaux (accusant un lourd déficit, accru depuis le 11 septembre) et qu'il sera nécessaire de reconstruire au plus vite le sud de Manhattan, ce sont les autres budgets qui pâtiront avec leur cortège de rues sales, de sans abris, de familles assistées au point que des entreprises et des familles songeront à nouveau à quitter la ville. 15400 emplois ont émigré vers le New Jersey en octobre, on ne sait pas si cette relocalisation est temporaire - causée par la destruction des bureaux - ou permanente ${ }^{18}$. Selon Felix Rohatyn, ancien ambassadeur des Etats-Unis à Paris qui avait participé à la reconstruction financière de la ville en 1976, «New York est plus en danger aujourd'hui et elle n'a pas les ressources nécessaires pour se relever seule ». Or après que plus de 600000 emplois eurent été éliminés entre 1969 et 1975, lorsque le maire de New York s'était tourné, en 1976, vers le Président Ford, républicain, il n'en avait reçu que cette réponse laconique « Drop dead » (allez vous faire voir ${ }^{19}$. Pendant des années, la ville avait souffert des coupes sombres qu'elle avait dû opérer dans ses services à ce moment-là. Selon Jeff Fagan qui dirige le centre d'étude de la violence à Columbia University, l'explosion des taux de criminalité des années 1980 est corrélée aux coupes dans le budget des écoles publiques qui ont suivi la banqueroute de 1975. Aujourd'hui, la qualité des écoles publiques est médiocre. Baisser leur budget, dit-il, c'est s'exposer aux mêmes risques en termes de reprise de la délinquance.

Le contexte n'est certes plus le même. L'assiette foncière est infiniment plus saine et les comptes tenus à jour. La ville a des dettes - pour chaque dollar d'impôt, dix neuf cents servent à rembourser la dette - mais elles ne sont pas de même nature que celles des années 1970 qui étaient incontrôlées. L'Etat de New York a autorisé la municipalité à émettre un emprunt à court terme de 2,5 milliards de dollars en attendant l'aide fédérale ${ }^{20}$. L'obtiendra-t-elle ? L'aide sera-t-elle à la mesure de ses attentes ? L'histoire américaine axée sur la compétition et sur l'individualisme entrepreneurial est cruelle envers ses villes. Après les émeutes de Los Angeles en 1982, un certain nombre de millions de dollars fédéraux devaient être affectés par l'administration Reagan et le Congrès à la reconstruction de la ville. Puis un ouragan détruisit une partie de la Floride, peuplée de retraités, électorat républicain et les fonds destinés à Los Angeles furent réaffectés à la Floride. Les hommes d'affaires qui s'étaient engagés à aider la cité dans une organisation appelée "Rebuild L.A.» se chamaillèrent et au bout de quelque temps, la clé fut mise sous la porte. Les quartiers brûlés sont restés couverts de cendres. Certes New York n'est pas Los Angeles et le Lower Manhattan n'est pas South Central. Mais lorsqu'il s'agit de dépenses publiques, mieux vaut être du côté majoritaire au Congrès et faire partie des amis du Président. Ceci justifie sans doute le choix avisé des 
New Yorkais en faveur du milliardaire Bloomberg qu'ils viennent d'élire maire de New York. Dès le 14 novembre, celui-ci a entrepris son pèlerinage pour quémander neuf milliards de dollars à Washington. Il ne fait aucun doute qu'il a été mieux reçu qu'Abraham Beame en 1975, d'une part parce qu'il est Républicain, bien qu'ancien démocrate, et dauphin de Giuliani et que la Maison Blanche mise sur lui pour l'emporter à New York aux prochaines élections, et d'autre part, parce que le sort de New York a ému fortement le pays qui se sent solidaire d'une ville brutalement éviscérée. Mais l'administration Bush s'est pour le moment opposée à cette aide ${ }^{21}$. Ce n'est qu'un début. Les marchandages ne font que commencer, selon un protocole bien rôdé22.

Le manque crucial d'argent, l'absence d'expérience politique et la menace continue de nouvelles attaques terroristes ne sont pas les seuls problèmes que le nouveau maire aura à résoudre. Le système fédéral lui-même, avec ses rites de méfiance institutionnalisés entre échelons de pouvoir, et avec son étanchéité chronique d'un service à l'autre se révèle particulièrement dysfonctionnel lorsqu'il s'agit de déployer des dispositifs de prévention contre les attaques terroristes sur le terrain local. Le 29 octobre 2001, Rudolph Giuliani a réclamé avec insistance une loi qui permettrait d'accroître de manière significative le volume d'informations échangées entre les administrations fédérales chargées de la sécurité et les administrations locales chargées de la loi et de l'ordre. Il a demandé, par exemple, la création d'un nouveau bureau du FBI, dirigé par un haut fonctionnaire (Tom Ridge, le nouveau coordonnateur de la sécurité intérieure ne dispose que d'une équipe restreinte et d'un budget symbolique) qui mettrait en application une telle loi et coordonnerait le partage de l'information. Le maire de New York n'est pas le seul à se plaindre devant la souscommission du Congrès chargée du terrorisme et de la sécurité dans le pays (ironie du sort, la réunion de cette sous-commission, prévue avant le 11 septembre, devait se dérouler dans les Twin Towers). Frank Keating, maire d'Oklahoma, évoque sa frustration après l'explosion de l'immeuble fédéral en 1995. "La réponse doit être nationale, et non fédérale» observe-t-il, indiquant par là que l'éparpillement des prérogatives dans un système fédéral nuit à l'efficacité et à la cohérence qui devrait être nationale. Lui-même fut un agent du FBI, alors marqué par une culture du secret cultivée par J. E. Hoover. "Mais nous ne sommes plus dans la même culture », ajoute-t-il, "et les agents du FBI sont plus diplômés que les anciens".

Avant le 11 septembre, en 1996, la mégalomanie de R. Giuliani avait été mise en cause lors de la création au World Trade Center d'un Bureau municipal chargé d'agir promptement en cas d'urgence (Office of Emergency Management). On sait ce qu'il en est advenu. Mais cette agence-bunker avait mis le doigt sur un certain nombre de dysfonctionnements. Or ils sont devenus encore plus apparents après le 11 septembre et le maire s'est plaint d'une «barrière visible» empêchant les échanges d'informations critiques entre le FBI et la police locale pendant cette crise extrême, alors que le premier, ajoutait-il, pourrait tirer avantage des connaissances des quartiers accumulées par la seconde. Lors du premier incident relatif à la maladie du charbon frappant la chaîne de télévision NBC dans la ville, la municipalité n'en a pas été immédiatement informée. Le FBI s'est montré plus efficace à mener des assauts contre le maire que contre les terroristes, observe l'éditorialiste du New York Times, F. Rich (27/10/2001). Il n'a transmis à la police locale que des feuilles avec des noms, action dérisoire puisque chacun sait que les terroristes opèrent sous des pseudonymes ${ }^{23}$. 
Or, si imparfaite soit l'action fédérale, cette demande locale peut s'avérer dangereuse pour les libertés, à l'aune de l'histoire américaine. La police locale, quoi qu'en dise R. Giuliani, a longtemps harcelé les minorités dans les Etats du sud et dans de nombreuses villes du Nord-Est. Confier à tel ou tel gouverneur la liberté d'appliquer le Patriot Act à sa manière pourrait conduire à mettre la plupart des téléphones sur écoute, à surveiller les échanges sur les sites Internet et à accumuler des renseignements sur la vie privée des citoyens. L'approbation de ces mesures par des juges élus localement ne serait pas une garantie. Même si l'époque n'est plus la même, le clonage du FBI dans cinquante Etats relève d'un scénario à la Orwell.

Retour au maelström : le manque de clairvoyance politique

Bien que considérée comme une ville-monde, New York continue à être territorialement localisée sur le territoire américain et son avenir demeure solidaire de celui du pays. La politique menée par Washington a nécessairement une incidence sur le sort de la ville. Or malgré un réaménagement des priorités, le moment des grandes remises en cause de la politique extérieure américaine qui a trop souvent soutenu des régimes autoritaires, négligé le tiers monde et soutenu Israël sans discontinuer ne semble pas venu à Washington. Comme le montrent les sondages précédemment cités, l'opinion n'est pas préparée à admettre la complexité des choix et la validité des options autres que militaires alors que les médias jouent sans discontinuer la carte belliciste. Alors que la plupart des Américains disent être très mal informés sur la France et que la visite de son Président ne fait pas deux lignes dans la presse nationale, comment s'attendre à ce qu'ils en sachent davantage sur le Tiers Monde et questionnent la politique étrangère des Etats-Unis? Le journaliste Ken Auletta observait qu'en 1988, les trois grandes chaînes offraient chacun 2000 minutes de couverture étrangère, en 2000, ce chiffre était tombé à 1200 . Désormais les nouvelles de l'étranger ne représentent plus que $9 \%$ d'une émission de 19 minutes en moyenne (la situation changera peut-être avec le 11 septembre). Dan Rather, l'archétype du présentateur de journal télévisé, dénonce "l'Hollywoodisation et la frivolité " des informations. Entre l'écrasement des dissidents chinois et le problème du ronflement chez les Américains, le ronflement gagne ${ }^{24}$. «Pourquoi nous haïssent-ils? » se demandentils interloqués? Or les effets pervers de la mondialisation sont connus : «Je crois que l'on doit penser aujourd'hui en termes d'effondrement social», admettait J. Wolfensohn, President de la World Bank quelques mois avant le 11 septembre, en avançant ses statistiques : 1.2 milliards de gens continuent à vivre avec moins de 1 dollar par jour et 1,3 milliards avec 2 dollars par jour. La plupart des hommes politiques ne saisissent pas que dans 20 ans le monde comptera 2 milliards de personnes de plus... Si on n'investit pas massivement dans le monde en développement, il n'y aura pas de paix dans vingt $a^{25}{ }^{25}$. Le non partage des pays riches produit un terreau propice à la violence, pour peu qu'il soit manipulé par des théocrates peu enclins à reconnaître leurs propres errements. Hans Magnus Enzensberger nous met en garde contre le retour des pulsions de mort et contre la logique d'automutilation. "Il est difficile d'ignorer... le degré d'autodestruction auquel les acteurs aspirent... le désir de naufrage est irrésistible » écrit-il, "jusque et y compris dans les sociétés prétendument ultra-développées » ${ }^{26}$.

L'un des impacts des désordres générés par la mondialisation consiste à brouiller les frontières entre les domaines de politiques intérieure et extérieure, ce qui amène à une "globalisation» de la contestation - mais également de la répression, puisque les politiques de sécurité effacent la distinction entre les dispositifs de sécurité interne et externe. Une militarisation des techniques utilisées par les forces de police et une 
«policisation » des interventions militaires urbaines sont déjà à l'œuvre. Pendant les désordres causés par la contestation des réunions de l'organisation du commerce mondial dans différentes villes, certains porte-paroles des ONG utilisaient le même langage que celui des minorités durablement installées dans la ville: demandes de reconnaissance, d'expression, de droits, de meilleur partage, de légitimité pour l'accès à une citoyenneté de plein droit (dans les trois acceptions proposées par T.S.

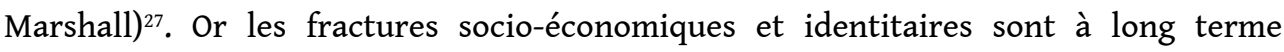
porteuses de violence, c'est à dire, selon la définition de $\mathrm{H}$. Nieburg, d'«actes de désorganisation, destruction, blessures, dont l'objet, le choix des cibles ou des victimes, les circonstances, l'exécution et/ ou les effets acquièrent une signification politique, c'est à dire, tendent à modifier le comportement d'autrui dans une situation de marchandage qui a des conséquences sur le système social $»^{28}$.

Les attaques du 11 septembre 2001 ne seront pas suivies de marchandages et de compromis. Le jeu est à somme nulle. On ne négocie pas avec al-Qaida.

Pourtant il serait faux de dire que rien ne sera plus pareil après le 11 septembre. Les choses ne changent pas aussi vite, surtout à Washington. Les échecs visibles ne sont pas produits par la confusion résultant d'un état de guerre mais sont les conséquences directes d'une mentalité qui s'est imposée le 20 janvier 2001 et qui favorise les intérêts privés, principalement pétroliers et texans, au détriment de l'intérêt public. Les négociations de l'administration Bush avec les talibans jusqu'en juillet 2001 en seraient une preuve avancée par quelques experts de même que les compromis envers les intérêts pharmaceutiques et la prévention envers la menace biochimique ou le soutien financier accordé aux cadres des compagnies aériennes mais non aux employés ou enfin les faveurs déployées envers ceux qui tirent profit des opérations militaires.

Une démocratie « disjonctive »

Dans les options prises en vue du renforcement de la sécurité, il apparait essentiel que les nouveaux pouvoirs de police dont se dotent la plupart des pays occidentaux ne soient pas détournés à des basses besognes de traque des habituels suspects et que les légitimes revendications des libertés soient prises au sérieux. C'est là le piège tendu ${ }^{29}$. Le sentiment d'insécurité impose un état de tension sans fin qui s'infiltre dans l'existence et la gâche. Progressivement, on se trouve entouré d'un nombre infini de systèmes de sécurité, censés protéger, mais qui ne font qu'augmenter notre malaise et diminuer le sentiment de sécurité. Comme c'est déjà le cas à New York, on est filmé à son insu, en entrant dans un musée. Des policiers, des vigiles, des agents secrets ou officiels se tiennent à l'entrée des cinémas, des théâtres, des écoles et des centres commerciaux. Y aura-t-il assez de gardes pour examiner tous ceux qui se rendent dans le métro ? Combien de temps faudra-t-il arriver à l'avance pour prendre un avion ou assister à un match afin que les fouilles puissent se faire? On ne peut que regretter que tant d'énergie soient utilisées pour la sécurité, alors qu'elle aurait pu l'être pour améliorer la créativité, la pensée ou la qualité de la vie. « Pour protéger la vie, on restreint les libertés et les droits individuels...Nous verrons de plus en plus de gens armés dans les rue des Etats-Unis, du Royaume Uni et du reste de l'Europe... Cette présence d'armes à feu aura des conséquences. Il suffira de dire: 'J'ai cru que c'était un terroriste'. Ce sera le premier résultat d'une vie marquée par la peur et la méfiance ${ }^{30}$. Qu'on le veuille ou non, le proche et le semblable deviennent des suspects en puissance en période d'inquiétude généralisée. Les murs qui, autrefois, enserraient la ville pour la protéger d'ennemis extérieurs, forment désormais un maillage dru, physique ou virtuel à l'intérieur même de la ville. Il 
en est de même de la pratique démocratique devenue «disjonctive ». Les caractéristiques ethniques et raciales deviennent des marqueurs grossiers pour identifier ceux qui font partie du club et ceux qui n'y ont pas accès. Or le juriste William O'Douglas observait un jour que les garanties pour les libertés ne s'appliquent pas d'elles-mêmes. C'est aux citoyens qu'il revient d'être vigilants. "Tout comme la nuit ne tombe pas d'un seul coup,ajoutait-il,il en va de même de l'oppression. Dans les deux cas, il y a d'abord un crépuscule au cours duquel rien ne change en apparence. Et c'est lors d'un tel crépuscule que nous devons tous prendre conscience du changement qui est dans l'air, aussi infime, soit-il, sous peine de succomber sans le vouloir aux ténèbres ${ }^{31}$.

Il faut rester très vigilant, et ne pas hésiter à protester si les excès se multiplient.

$C^{\prime}$ est peut-être là ce que nos démocraties ont à dire : les garanties pour les libertés ne s'appliquent pas d'elles-mêmes et c'est aux citoyens qu'il revient de les protéger. Nous avons tous beaucoup à perdre - la volonté de vivre ensemble, la rencontre souhaitée avec l'Autre non connu - ces biens si fragiles qui semblent aujourd'hui particulièrement menacés.

\section{NOTES}

1. . James Holton, « Disjunctive Democracies », non publié, Conférence Democracy, Violence and Cities, New Segregations and changes in Public Space, Irvine, University of California, juin 2001.

2. . The New Republic, 24 octobre 2001.

3. . Le dispositif du renseignement inclut également 50000 personnes affiliées aux armées et disposant d'un budget annuel de 11 milliards de dollars. Isnard Jacques, « George Bush impose le mutisme à ses services de renseignement », Le Monde, 27 septembre 2001.

4. . Risen James, « How, and How Not, to Fix the F.B.I. », New York Times, 9 septembre 2001.

5. . Ainsi, plus d'un million d'armes se sont vendues au cours des trois mois qui ont suivi les attaques, selon les statistiques du FBI qui doit procéder à des contrôles d'identité lors de ces transactions. Même si les Américains disent faire confiance à l'équipe gouvernementale, ils préfèrent se protéger contre des ennemis énigmatiques. Les ventes ont été particulièrement élevées à New York, à Washington et en Floride. 6. . Washington Post/ABC News auprès de 759 adultes avec une marge d'erreur de plus ou moins $4 \%$.

7. . Voir The Economist, 1er décembre 2001 et l'International Herald Tribune du 30 novembre 2001.

8. . Elias Norbert, « Les pêcheurs dans le Maelström », Engagement et distanciation, Paris, Fayard, 1993, chap. 2. Je remercie P.J. Simon d'avoir attiré mon attention sur la nouvelle d'Elias.

9. . Traub James, « No-Fun City », New York Times Magazine, 4 novembre 2001. 
10. . Selon R. Reich, ancien ministre de l'emploi, le bilan en octobre 2001 se solde par la perte de 439000 emplois dans le secteur privé : pour l'hôtellerie, la perte est de 46000 , pour les commerces de 81000 , pour les compagnies aériennes de 42000 . Le taux de chômage chez les Noirs est monté à 9,7\%, soit 2,3 de plus que l'année précédente (2,2 pour les Latinos) Cf. Reich Robert, « Lost Jobs, Ragged Safety Net », New York Times, 12 novembre 2001.

11. . L'expression vient de Paul Goldberger, critique architectural new yorkais. Il considère que les Tours Jumelles comme le premier immeuble martyr des Etats-Unis. Eric Darton, auteur de Divided We Stand : A Biography of New York's World Trade Center, ajoute que ces tours « étaient quelque chose que nous avions envie de refuser, et maintenant nous donnerions n'importe quoi pour les avoir encore "...

12. . Body-Gendrot Sophie, The social control of cities? A comparative perspective, Oxford, Blackwell, 2000.

13. . NYPD, décembre 2001.

14. . Kauffmann Sylvie, «A New York, maintenant plus que jamais », Le Monde, 14 novembre 2001.

15. . Frau-Meigs Divina, Médiamorphoses américaines dans un espace privé unique au monde, Paris, Economica, 2001.

16. . Reich, op.cit.

17. . McEnroe John, « J'ai voulu y aller », Le Monde, 27 septembre 2001.

18. . Peterson Iver, «Wall St. Jobs Are Migrating to New Jersey After Attack », New York Times, 17 novembre 2001. L'actualité évolue si vite que certaines des informations seront obsolètes au moment de la publication, nous prions les lecteurs de nous en excuser.

19. . Body-Gendrot Sophie, Ville et violence. L'irruption de nouveaux acteurs, Paris, PUF, 1993.

20. . Purnick Joyce, « Recalling the 70's, Warily », New York Times, 11 novembre 2001.

21. . La commission d'appropriation budgétaire du Capitole a repoussé la demande de New York par trente trois voix contre trente et une.

22. . Ainsi, sous la pression de Washington, la ville de New York a dû accepter que les quatre entreprises qui, depuis six semaines, déblayaient le « point zéro » soient remplacées par la firme milliardaire Bechtel, laquelle avait largement contribué à la campagne présidentielle.

23. . L'ex-chef de la police new yorkaise, Bernard Kerik, abonde en ce sens. Il lui faut attendre qu'une information-clé passe par le filtre d'une commission, puis d'un certain nombre de juridictions et de personnes avant de la recevoir, et encore, sous réserve. Il rappelle qu'après l'attaque, alors qu'il descendait avec le maire le long du West Side, il ne savait pas à qui s'adresser pour que tout l'espace aérien autour du World Trade devienne inaccessible et protégé.

24. . Auletta Ken, «Battle Stations », The New Yorker, 10 décembre 2001, pp. 60-61. 25. . Cohen Richard, « Growing up and Getting Practical Since Seattle », New York Times, 24 novembre 2000.

26. . Enzensberger Hans Magnus, « Retour du sacrifice humain », Le Monde, 27 septembre 2001, p. VII.

27. . Body-Gendrot Sophie, Villes. La fin de la violence ? Paris, Presses de Sciences Po, 2001, chap. 1.

28. . Nieburg H., Political Violence : the Behavioral Process, New York, St Martin's Press, 1969, p. 13. 
29. . Grossman David, « Cette terreur qui corrompt tout », Le Temps, cité par le Courrier International, 570, 4-10 octobre 2001, p. 47.

30. . Ibid

31. . Hentoff Nat, «L'ombre du maccarthysme plane à nouveau », Village Voice, cité par le Courrier International, 570, 4-10 octobre 2001, p. 41.

\section{RÉSUMÉS}

Dans cet article, Sophie Body-Gendrot analyse les réactions à New-York face aux attentats et montre quand, pourquoi et comment les hommes politiques sont prêts à saisir l'opportunité de l'impact émotionnel des attentats pour relancer des politiques mises à l'écart ou refusées précédemment en analysant la position et les discours du maire de New-York Rudolph Giuliani. L'auteur rediscute par ailleurs la question de l'équilibre ente sécurité et libertés et souligne comment, à la faveur d'un développement de la méfiance envers son semblable se créé ainsi des clivages entre citoyens, à l'intérieur même d'une ville comme New-York.

The author analyses the New York reactions to the terrorist attacks and shows when, why and how politicians were ready to seize the emotional impact opportunity to re-launch politics previously put aside or refused by analysing the position and speeches of Rudolph Giuliani, New York's mayor. The author furthermore discusses the equilibrium between security and liberty and highlights how cleavages can arise between citizens of a same city, as a consequence of the development of the mistrust of one's fellow man.

\section{INDEX}

Index géographique : Etats-Unis

Mots-clés : villes/ sociologie urbaine 